\title{
Exiguobacterium Sp. HA2, isolated from the Ilam Mountains of Iran
}

\author{
Reza Heidari ${ }^{1}$, Mostafa Akbariqomi ${ }^{1}$, Gholamreza Tavoosidana ${ }^{1,}$ Garshasb Rigi $^{2,3^{*}}$
}

${ }^{1}$ Department of Molecular Medicine, School of Advanced Technologies in Medicine, Tehran Uni versity of Medical Sciences, Tehran, Iran.

${ }^{2}$ Department of Genetics, Faculty of Basic Science, Shahrekord University, Shahrekord, Iran.

${ }^{3}$ Department of Industrial Biotechnology, Research Institute of Biotechnology, Shahrekord University, Shahrekord, Iran

*Corresponding author: Dr. Garshasb Rigi, Department of Genetics, Faculty of Basic Science, Shahrekord University, Rahbar Blvd, P O Box 115, Shahrekord 881863 4141, Iran, Email: garshasbiotech@sku.ac.ir 


\begin{abstract}
A motile, Gram-stain-positive, rod-shaped, non-sporing, tolerate up to $5 \% \mathrm{NaCl}$, grew at $0-25{ }^{\circ} \mathrm{C}$, designated Exiguobacterium sp. HA2 was isolated from the soil of the Ilam Mountains of Iran during October 2016. The major isoprenoid quinone is MK-7 and in the smaller amount are MK6 and MK-8. Polar lipids included diphosphatidylglycerol, phosphatidylglycerol, phosphatidylserine, phosphatidylinositol, phosphatidylethanolamine. Major fatty acids (>10\%) are iso $\mathrm{C}_{13: 0}$, iso $\mathrm{C}_{15: 0}$ and $\mathrm{C}_{16: 0}$. The bacterial cell wall peptidoglycan layer was lysine-glycine. The $16 \mathrm{~S}$ rRNA sequence was analyzed at the phylogenetic levels. Also, A supplemental comparison was made between five other genes including $c s p, g y r \mathrm{~B}, h s p 70, r p o \mathrm{~B}$, and $c i t \mathrm{C}$. According to the results of genotypic and phenotypic characteristics, the strain was categorized in the genus Exiguobacterium. This bacterium had the closest relation with Exiguobacterium undae, and thus was dubbed Exiguobacterium sp. HA2. The different in the Phenotypic, functional characteristics and phylogenetic indicated Exiguobacterium sp. HA2 can be regarded as representing considered a novel species within the genus Exiguobacterium.
\end{abstract}

Keywords: Psychrotolerant, Exiguobacterium, novel species, Polyphasic taxonomy

Author Notes: The GenBank/EMBL/DDBJ accession number for the 16S rRNA gene, gyrB, citC, rpoB, hsp70, and csp sequence of strain Exiguobacterium sp. HA2 are MK272780, KX574228, MH370480, MH378445, MH378775, and MH379139, respectively. 


\section{Introduction:}

The genus of Exiguobacterium includes psychrophilic, thermophilic and mesophilic strains and species [1]. The Exiguobacterium genus is anaerobic, gram-positive bacteria of low $\mathrm{G}+\mathrm{C}$ contents. This bacterium has been repeatedly isolated from the ancient Siberian sediments, as well as environments such as Greenland glacial, the spa springs (Yellowstone National Park), and food processing environments. Therefore, the isolation sources of this bacterium are totally different and often in extreme environments [2]. Accordingly, each of these species or strains appears to have specifically and exclusively adapted to the environments. The diversity in their genome has been responsible for their adaptation to different conditions [3].

This genus was divided into two groups according to the $16 \mathrm{~S}$ rDNA sequence and genomic sequence; group I is the psychrophilic Exiguobacterium, and group II thermophilic [4]. Many strains of Exiguobacterium have unique properties that make them suitable for applications in biotechnology, detergent industry, sewage treatment, and agriculture [5]. Psychrophilic microorganisms have been described that are capable of growing at $<20^{\circ} \mathrm{C}[6]$.

In the present study, Exiguobacterium was isolated from the soil samples collected from the Ilam Mountains (one of Iran's cold provinces), and its genotypic and phenotypic characteristics were assessed and identified.

\section{Isolation, cultivation conditions and maintenance of strain}

Exiguobacterium sp. HA2 has been isolated from soil samples by cold enrichment method. The soil samples were collected from the top layer of soil $(5 \mathrm{~cm})$ from an area in Ilam $\left(33^{\circ} 45^{\prime} 30.32^{\prime \prime}\right.$ $\left.\mathrm{N}, 46^{\circ} 11^{\prime} 51.33^{\prime \prime} \mathrm{E}\right)$. The soil sample was store at cold room at $4{ }^{\circ} \mathrm{C}$ for $72 \mathrm{~h}$ before isolation. For isolation, $10 \mathrm{~g}$ of soil sample was added in $50 \mathrm{ml}$ sterile water, and then the suspension was incubated at $4{ }^{\circ} \mathrm{C}$ for $60 \mathrm{~min}$. The strain was isolated on Tryptone Soy Agar (TSA; Difco Laboratories, Detroit, MI, USA) at $4^{0} \mathrm{C}$ for 5 days. The isolated bacterium was stored at $-80^{\circ} \mathrm{C}$ in TSB (Tryptone Soya Broth) supplemented with 25\% glycerol for cryoprotectection.

\section{Genomic characterization}

Genomic DNA was extraction and purification using the THP(Triton/Heat/Phenol) slight modification of protocol [7]. Also, Phylogenetic comparisons were performed between the mentioned new strain and sequences of five other genes including csp (universal major cold shock 
protein), gyrB (gyrase subunit B), hsp70 (Class I-heat shock protein-chaperonin), rpoB (DNAdirected RNA polymerase beta subunit), and citC (isocitrate dehydrogenase). To confirm bacterial identification, we amplified the genes (16s rRNA, $\operatorname{sp}$, gyrB, $h \operatorname{sp} 70, \operatorname{rpoB}, \operatorname{cit} C$ ) directly by using the universal primers for Exiguobacterium (Table1). The annealing temperatures were respectively 56, 53, for $c s p, r p o \mathrm{~B}$, and $49^{\circ} \mathrm{C}$ for $c i t \mathrm{C}$ and $g y r \mathrm{~B}$, and $47^{\circ} \mathrm{C}$ for $h s p 70$. The PCR amplified products were purified using QIAquick Kit (PCR Purification Kit, Qiagen, Valencia, CA, USA) and then sequenced [8]. The TOPO TA Cloning Kit (Life Technologies) was used for the cloning and sequencing of the amplicons of other genes. The Qiagen Plasmid Kit (Qiagen, Valencia, CA, USA) was then used to extract the obtained clones. An ABI 373a DNA sequencer was used for the analysis of the products of cycle sequencing performed by a Perkin-Elmer 9600 thermal cycler and an ABI Dye Terminator Chemistry (PE Applied Biosystems).

Genotypic analyses were conducted to clarify the phylogenetic relationship between the new strain and the most relevant set of five reference strains. The sequence analysis of the 16S rRNA gene indicated that new strain was categorized in the genus Exiguobacterium (Fig. 1). The sequence was deposited in GenBank with the accession number KT967971. The sequences of gyrB, citC, rpoB, $h s p 70$, and csp, also were deposited in GenBank with the accession numbers of KX574228, MH370480, MH378445, MH378775, and MH379139, respectively. Phylogeny analysis of other genes was performed to confirm identification the phylogenetic relationships of this strain within Exiguobacterium. Phylogenetic trees constructed based on gyrB (Fig. 2), citC (Fig. 3), rpoB (Fig. 4), $h s p 70$ (Fig. 5), and csp (Fig. 6) sequences of the following genes. Analysis of the 16S rRNA gene sequences indicates that HA2 had a high level of similarity to SH3 and undae (96\%). Also, the alignment and comparison sequence of $g y r \mathrm{~B}, \operatorname{cit} \mathrm{C}$, rpoB, hsp70, $\operatorname{ssp}$ of $\mathrm{HA} 2$ showed highest similarity to Exiguobacterium undae. Analysis of the gene sequences of HA2 with other members of the genus Exiguobacterium indicated that similarities with phylogenetic neighbours were in the range $60-100 \%$.

Using the MEGA5.0 program, we constructed Phylogenetic trees based on the neighbor-joining and distance methods. The phylogenetic tree was reconstructed with generated by the maximumlikelihood method and the reliability of each node was assessed with 1000 bootstrap test replications. The scale bar represents 0.1 substitution per nucleotide position. The roots of the trees were determined using those genes from Bacillus. 


\section{Morphology, physiology and biochemical characterization}

The optimum temperature required for the growth of Exiguobacterium sp. HA2 was $15^{0} \mathrm{C}$. A colony size desirable for scanning electron microscopy (SEM) was reached by incubating inoculated R2A plates at $4^{0} \mathrm{C}$ for 28 days. Samples for scanning electron microscopy (SEM) studies were prepared according to the previously published method [9]. Gram-staining was performed as described previously [10]. Minimum and maximum temperatures for growth was investigated on $\mathrm{R} 2 \mathrm{~A}$ agar after incubation for 5 days at -4 to $30^{\circ} \mathrm{C}\left(-4,0,4,10,15,20,25,30^{\circ} \mathrm{C}\right)$. Tolerance to $\mathrm{NaCl}$ was determined by incubating $\mathrm{R} 2 \mathrm{~A}$ broth supplemented with $\mathrm{NaCl}(0-10 \%, \mathrm{w} / \mathrm{v}$, at $0.5 \%$ intervals) concentrations in $1.5 \mathrm{ml}$. The $\mathrm{pH}$ range was evaluated using $\mathrm{R} 2 \mathrm{~A}$ broth containing various $\mathrm{pH}$ 6-10 (at intervals of $0.5 \mathrm{pH}$ unit) prior to autoclaving using appropriate buffers [11]. Presence of spores was evaluated by staining with malachite green. API 20NE, API ID 32GN and API ZYM (bioMerieux) were used for analyzing the production of different enzymes. Fatty acid extraction and analysis was determined by gas chromatography (GC) as described previously [12]. Antibiotic sensitivity testing was performed using standard methods [13]. The culture's ability to assimilate different carbon compounds was assessed using minimal medium $\left[\mathrm{K}_{2} \mathrm{HPO}_{4} 2 \%(\mathrm{w} / \mathrm{v})\right.$; $\mathrm{KH}_{2} \mathrm{PO}_{4} 0.5 \%(\mathrm{w} / \mathrm{v})$; agar $\left.1 \%(\mathrm{w} / \mathrm{v})\right]$. The analysis of chemotaxonomic markers was performed through cell wall amino acids [14], polar lipids [15], peptidoglycan structure [16] and isoprenoid quinones [17]. E. sibiricum DSM 17290, E. antarcticum DSM 14480T, E. oxidotolerans JCM 12280T, E. acetylicum DSM 20416T, E. undae DSM 14481T, and were used as reference strains in morphological and biochemical assessments and fatty acids identification.

Total the membrane lipoquinones and polar lipids were extracted and separated according to the previous described [18] phospholipids were visualized by staining with molybdenum blue spray (Sigma). The peptidoglycan was isolated and analyzed using a method adapted from Schleifer and Kandler [14]. after hydrolysis the cells, total-cell sugars were analysis by TLC on cellulose plates according to the previous methods [19]. The major isoprenoid quinone is MK-7 and in the smaller amount are MK-6 and MK-8. Polar lipids compositions including diphosphatidylglycerol, phosphatidylglycerol, phosphatidylserine, phosphatidylinositol, phosphatidylethanolamine. Major fatty acids (>10 \%) are iC13:0, iC15:0 and C16:0; minor components are listed in Table 2. The peptidoglycan type is lysine-glycine. 
Cells of strain HA2 were Gram-positive, non-sporing, motile, facultatively anaerobic, rod-shaped, approximately $0.8-1 \mu \mathrm{m}$ in width and $1.5-2 \mu \mathrm{m}$ in length (Fig.7). Growth occurred at between 0 and $25^{\circ} \mathrm{C}$ (optimally, $15^{\circ} \mathrm{C}$ ), $\mathrm{pH}$ grows at $\mathrm{pH}$ 6-10 (optimally, 7.0) and in the presence of 0-12 (w/v) $\mathrm{NaCl}$ with an optimum of approximately 3 (w/v) $\mathrm{NaCl}$. positive in tests for oxidase, Gelatinase, b-galactosidase, DNase, catalase, Caseinase, Phosphatase, Lysine decarboxylase, but are negative for, urease and $\mathrm{H}_{2} \mathrm{~S}$ production and for the indole test. Exiguobacterium sp. HA2 utilize D-Fructose, D-Galactose, D-Mannose, L-Rhamnose, Cellobiose, D-Lactose, Maltose, Starch, Amygdalin, Arbutin, Glycogen, Citrate utilization. Strain HA2 was susceptible to Amikacin $(30 \mu \mathrm{g})$, Amoxicillin $(30 \mu \mathrm{g})$, Clindamycin $(25 \mu \mathrm{g})$, Colistin $(10 \mu \mathrm{g})$, Doxycycline $(25 \mu \mathrm{g})$, Co-trimoxazole $(25 \mu \mathrm{g})$, Nalidixic acid $(30 \mu \mathrm{g})$, Norfloxacin $(10 \mu \mathrm{g})$, Nitrofurantoin $(300 \mu \mathrm{g})$, Sulfamethoxazole $(50 \mu \mathrm{g})$, tobramycin $(15 \mu \mathrm{g})$, lomefloxacin $(30 \mu \mathrm{g})$, roxithromycin $(30 \mu \mathrm{g})$, ciprofloxacin $(30 \mu \mathrm{g})$, lincomycin $(15 \mu \mathrm{g})$, cefotaxime $(30 \mu \mathrm{g})$, cefazolin $(30 \mu \mathrm{g})$, kanamycin $(30 \mu \mathrm{g})$, novobiocin $(30 \mu \mathrm{g})$, chloramphenicol $(30 \mu \mathrm{g})$, ampicillin $(25 \mu \mathrm{g})$, tetracycline $(30 \mu \mathrm{g})$, streptomycin $(25 \mu \mathrm{g})$, erythromycin $(15 \mu \mathrm{g})$, bacitracin $(10 \mu \mathrm{g})$, gentamicin $\mathrm{G}(30 \mu \mathrm{g})$, polymyxin $\mathrm{B}(50 \mu \mathrm{g})$, oleandomycin $(15 \mu \mathrm{g})$, spectinomycin $(100 \mu \mathrm{g})$, rifampicin $(25 \mu \mathrm{g})$ and carbenicillin $(100 \mu \mathrm{g})$. but resistant to ceftriaxone $(30 \mu \mathrm{g})$ norfloxacin $(10 \mu \mathrm{g})$, gentamycin $(10 \mu \mathrm{g})$. The phenotypic features and biochemical profile of HA2 compare with other reference strains are description and illustrated in Table 3.

\section{Description of Exiguobacterium sp. HA2}

Phenotypic evaluations were also conducted to clarify the phenotypic distinguishability of the new isolates from each other and the reference Exiguobacterium strains. Scanning electron microscope images of the strain are shown in Fig. 7. Cells are Gram-positive, rod-shaped (2-3mm), aerobic, non-spore-forming. As presented in Table 1, surface colonies are bright, yellowish orange, convex, entire and shiny. Growth occurs at 0 to $25^{\circ} \mathrm{C}$; optimal temperature for growth about $15^{\circ} \mathrm{C}$. Tolerate to $5 \% \mathrm{NaCl}$, grow at $\mathrm{pH}$ 6-10, are positive in tests for oxidase, Gelatinase, b-galactosidase DNase, catalase, Caseinase, Phosphatase, amylase, lipase and protease. Exiguobacterium. sp. HA2 utilize D- glucose, sucrose, starch, Trehalose, Raffinose, Cellulose, Amygdalin, Arbutin, Glycogen, DSorbitol, Citric acid, Lactic acid. Cells are sensitive to the following antibiotics ( $\mu \mathrm{g})$ : Amikacin (30) Amoxicillin (30) Clindamycin (25) Colistin (10) Doxycycline (25) Co-trimoxazole (25) 
Nalidixic acid (30) Norfloxacin (10) Nitrofurantoin (300) Sulfamethoxazole (50). The peptidoglycan type is lysine-glycine. The major isoprenoid quinone is MK-7 and in the smaller amount are MK-6 and MK-8. Polar lipids included diphosphatidylglycerol, phosphatidylglycerol, phosphatidylserine, phosphatidylinositol, phosphatidylethanolamine. Major fatty acids (>10\%) are iC13:0, iC15:0 and C16:0; minor components are listed in Table 2. Isolated from a soil from Ilam, Iran.

\section{Conflicts of interest}

The authors declare that there are no conflicts of interest.

\section{Ethical statement}

This article does not contain any studies with animals performed by any of the authors. 


\section{References:}

1. Yadav, A.N., et al., Psychrotrophic microbiomes: molecular diversity and beneficial role in plant growth promotion and soil health, in Microorganisms for green revolution. 2018, Springer. p. 197-240.

2. da Costa, W.L.O., et al., Functional annotation of hypothetical proteins from the Exiguobacterium antarcticum strain $B 7$ reveals proteins involved in adaptation to extreme environments, including high arsenic resistance. PloS one, 2018. 13(6): p. e0198965.

3. Vishnivetskaya, T.A., S. Kathariou, and J.M. Tiedje, The Exiguobacterium genus: biodiversity and biogeography. Extremophiles, 2009. 13(3): p. 541-555.

4. Crapart, S., et al., Exiguobacterium profundum sp. nov., a moderately thermophilic, lactic acid-producing bacterium isolated from a deep-sea hydrothermal vent. International journal of systematic and evolutionary microbiology, 2007. 57(2): p. 287-292.

5. Heydari, R., et al., A statistical approach to the optimization of cold-adapted amylase production by Exiguobacterium sp. SH3. Starch-Stärke, 2012. 64(12): p. 955-963.

6. Morita, R.Y., Psychrophilic bacteria. Bacteriological reviews, 1975. 39(2): p. 144.

7. Akbariqomi, M., et al., Evaluation and statistical optimization of a method for methylated cell-free fetal DNA extraction from maternal plasma. Journal of assisted reproduction and genetics, 2019. 36(5): p. 1029-1038.

8. Eden, P.A., et al., Phylogenetic analysis of Aquaspirillum magnetotacticum using polymerase chain reactionamplified 16S rRNA-specific DNA. International journal of systematic and Evolutionary microbiology, 1991. 41(2): p. 324-325.

9. Jin, L., et al., Streptomyces inhibens sp. nov., a novel actinomycete isolated from rhizosphere soil of wheat (Triticum aestivum L.). International journal of systematic and evolutionary microbiology, 2019. 69(3): p. 688-695.

10. Doetsch, R., Determinative methods of light microscopy. Manual of methods for general bacteriology, 1981: p. 21-33.

11. Breznak, J.A. and R.N. Costilow, Physicochemical factors in growth. Methods for General and Molecular Microbiology, 2007: p. 309-329.

12. Kim, I.-G., et al., Exiguobacterium aestuarii sp. nov. and Exiguobacterium marinum sp. nov., isolated from a tidal flat of the Yellow Sea in Korea. International journal of systematic and evolutionary microbiology, 2005. 55(2): p. 885-889.

13. Smibert, R., Phenotypic characterization. Methods for general and molecular bacteriology, 1994.

14. Schleifer, K.H. and O. Kandler, Peptidoglycan types of bacterial cell walls and their taxonomic implications. Bacteriological reviews, 1972. 36(4): p. 407.

15. Minnikin, D., M. Collins, and M. Goodfellow, Fatty acid and polar lipid composition in the classification of Cellulomonas, Oerskovia and related taxa. Journal of Applied Bacteriology, 1979. 47(1): p. 87-95.

16. Schleifer, K.H., 5 Analysis of the chemical composition and primary structure of murein, in Methods in microbiology. 1985, Elsevier. p. 123-156.

17. Collins, M., et al., Chemotaxonomic Study of an Alkalophilic Bacterium, Exiguobacterium aurantiacum gen. nov., sp. nov. Microbiology, 1983. 129(7): p. 2037-2042.

18. Xu, X.-W., et al., Haloferax larsenii sp. nov., an extremely halophilic archaeon from a solar saltern. International journal of systematic and evolutionary microbiology, 2007. 57(4): p. 717-720.

19. Staneck, J.L. and G.D. Roberts, Simplified approach to identification of aerobic actinomycetes by thin-layer chromatography. Applied microbiology, 1974. 28(2): p. 226-231.

20. Rodrigues, D.F., et al., Characterization of Exiguobacterium isolates from the Siberian permafrost. Description of Exiguobacterium sibiricum sp. nov. Extremophiles, 2006. 10(4): p. 285-294.

21. Frühling, A., et al., Exiguobacterium undae sp. nov. and Exiguobacterium antarcticum sp. nov. Int J Syst Evol Microbiol, 2002. 52(Pt 4): p. 1171-1176. 
Table 1. Primers used for PCR amplification for Exiguobacterium genus analysis.

\begin{tabular}{|c|c|c|}
\hline Gene amplified & Sequence $\left(5^{\prime}-3^{\prime}\right)$ & Fragment size (bp) \\
\hline 16s rRNA & & $1506 \mathrm{bP}$ \\
\hline $16 s-F$ & AGG GTT GCG CTC GTT G & \\
\hline $16 s-\mathrm{R}$ & AAG GAG GTG WTC CAR CC & \\
\hline DNA Gyrase-beta subunit & & $930 \mathrm{bp}$ \\
\hline $\operatorname{gyr} B-\mathrm{F}$ & AAA CGT CCG GGT ATG TAT ATC GGA TCG AC & \\
\hline gyrB-R & CGG CGG CTG SGC AAT RTA SAC GTA & \\
\hline $\begin{array}{l}\text { ClassI-heat shock protein- } \\
\text { chaperonin }\end{array}$ & & $1453 \mathrm{bp}$ \\
\hline hsp70-F & CCC GAA TTC GGT AHA GTA AAA TGG TTY AAC KC & \\
\hline hsp70-R & CCC GGA TCC GGT TAC GTT ASC WGC TKS HGG DCC & \\
\hline $\begin{array}{l}\text { DNA-directed RNA polymerase } \\
\text { beta subunit }\end{array}$ & & $1072 \mathrm{bp}$ \\
\hline rpoB-F & CGA ACA TGC AAC GTC AGG C & \\
\hline rрoв-R & ACA TCY TCY TCA CGN GCA CC & \\
\hline Isocitrate dehydrogenase & & $1163 \mathrm{bp}$ \\
\hline citC-F & GGD GAY GGM ACW GGW CCW GAY ATT TGG & \\
\hline citC-R & AAT TCW GAA CAT TTM ACT TCT GT & \\
\hline $\begin{array}{l}\text { Universal major cold shock } \\
\text { protein }\end{array}$ & & $201 \mathrm{bp}$ \\
\hline CSP-U5 & CCC GAA TTC GGT AHA GTA AAA TGG TTY AAC KC & \\
\hline CSP-U3 & CCC GGA TCC GGT TAC GTT ASC WGC TKS HGG DCC & \\
\hline
\end{tabular}


Table 2. Fatty acid composition of the Siberian permafrost isolates and the type strains of Exiguobacterium

\begin{tabular}{|c|c|c|c|c|c|c|c|}
\hline & HA2 & $7-3^{b}$ & $255-15^{b}$ & $190-11^{\mathrm{b}}$ & E. undae ${ }^{\mathrm{a}}$ & E. antarcticum ${ }^{a}$ & E. aurantiacum ${ }^{\mathrm{a}}$ \\
\hline $\mathrm{iC}_{11: 0}$ & & & & & & & 2 \\
\hline $\mathrm{iC} 12: 0$ & 3 & 2 & 2 & 2 & 2 & 3 & 3 \\
\hline $\mathrm{C}_{12: 0}$ & 1 & 1 & & 1 & & 1 & 2 \\
\hline iC13:0 & 11 & 9 & 13 & 8 & 9 & 12 & 18 \\
\hline $\mathrm{AiC}_{13: 0}$ & 8 & 11 & 15 & 10 & 9 & 11 & 12 \\
\hline $\mathrm{iC}_{14: 0}$ & 9 & 1 & 1 & 1 & 2 & 1 & \\
\hline $\mathrm{C}_{14: 0}$ & 2 & 3 & 1 & 2 & 2 & 2 & 3 \\
\hline $\mathrm{iC}_{15: 0}$ & 11 & 13 & 12 & 13 & 10 & 11 & 4 \\
\hline aiC15:0 & 4 & 3 & 4 & 3 & 3 & 2 & \\
\hline $\mathrm{C}_{16} \mathrm{~s} 1-11 \mathrm{c}$ & 9 & 8 & 3 & 7 & 8 & 18 & 10 \\
\hline $\mathrm{iC}_{16: 0}$ & 3 & 2 & 2 & 2 & 2 & & \\
\hline $\mathrm{C}_{16}: 1-5 \mathrm{c}$ & & 1 & & 1 & & & \\
\hline $\mathrm{C}_{16}: 1-7 \mathrm{c}$ & & 1 & & 1 & 7 & 3 & \\
\hline $\mathrm{C}_{16: 0}$ & 16 & 17 & 20 & 12 & 17 & 13 & 27 \\
\hline $\mathrm{C}_{17: 1-10 \mathrm{c}}$ & 4 & 2 & 2 & 3 & 2 & 3 & \\
\hline iC17:0 & 5 & 9 & 12 & 8 & 7 & 5 & 6 \\
\hline aiC $17: 0$ & 3 & 3 & 3 & 3 & 2 & & \\
\hline $\mathrm{C}_{18}: 1-9 \mathrm{c}$ & & 2 & 1 & 2 & 3 & 6 & 2 \\
\hline $\mathrm{C}_{18: 1-7 \mathrm{c}}$ & 2 & 2 & 1 & 3 & 3 & & \\
\hline $\mathrm{C}_{18: 0}$ & 5 & 4 & 5 & 3 & 6 & 5 & 5 \\
\hline
\end{tabular}

Strains: 1, Exiguobacterium sp. HA2; 2, Exiguobacterium strains 7-33; 3, Exiguobacterium strains 255-15; 4, Exiguobacterium sp. 190-11; 5, E. undae DSM 14481T; 6, E. antarcticum DSM 14480T; 7, E. aurantiacum DSM 6208T.

Only values $>1 \%$ are indicated; values $\geq 5 \%$ are given in bold aData obtained from Rodrigues et al. (2006) [20]

bData obtained from Fruhling et al. (2002) [21] 
Table 3. Phenotypic features distinguishing strain Exiguobacterium sp. HA2 from the six most closely related species of the genus Exiguobacterium

\begin{tabular}{|c|c|c|c|c|c|c|c|}
\hline Characteristic & 1 & 2 & 3 & 4 & 5 & 6 & 7 \\
\hline Source & Soil & Microbial mat & Siberian & Garden pond & Glacial water & Drainage from & $\begin{array}{c}\text { Creamery } \\
\text { waste }\end{array}$ \\
\hline Colony size $(\mathrm{mm})$ & $2-3$ & $2-3$ & $2-3$ & $2-4$ & $2-4$ & $1-5$ & $2-5$ \\
\hline Colony shape & Round & Round & Round & Round & Round & Round & Irregular \\
\hline Colony colour & $\begin{array}{c}\text { Yellowish } \\
\text { orange }\end{array}$ & Orange & Orange & Orange & $\begin{array}{c}\text { Yellowish } \\
\text { orange }\end{array}$ & Orange & $\begin{array}{c}\text { Yellowish } \\
\text { orange }\end{array}$ \\
\hline \multicolumn{8}{|c|}{ Growth temperature (uC) } \\
\hline 2.5 & + & - & + & - & W & W & - \\
\hline 5 & + & - & + & - & + & + & - \\
\hline 37 & - & + & + & + & - & + & + \\
\hline $\begin{array}{l}\text { Maximum growth } \\
\text { temperature }\left({ }^{\circ} \mathrm{C}\right)\end{array}$ & 25 & 41 & 40 & 41 & 30 & 40 & 37 \\
\hline Gelatinase & + & + & + & + & - & + & + \\
\hline DNase & + & + & - & + & - & + & + \\
\hline Caseinase & + & + & + & + & - & + & + \\
\hline Phosphatase & + & + & + & - & + & + & + \\
\hline Lysine decarboxylase & + & - & + & + & + & - & - \\
\hline Arginine decarboxylase & + & + & + & + & + & - & - \\
\hline Tryptophan eamination & + & + & + & - & - & + & + \\
\hline Aesculin hydrolysis & + & + & + & + & - & + & + \\
\hline Starch hydrolysis & + & + & + & + & - & + & + \\
\hline Citrate utilization & - & - & + & - & + & + & + \\
\hline Malonate utilization & + & - & - & - & + & - & - \\
\hline $\begin{array}{l}\text { Reduction of nitrate to } \\
\text { nitrite }\end{array}$ & - & - & - & - & + & - & - \\
\hline Methyl red test & + & + & - & + & 2 & + & + \\
\hline
\end{tabular}

\section{Acid production from:}

\begin{tabular}{|l|c|c|c|c|c|c|}
\hline Melibiose & - & - & + & - & - & - \\
\hline D-Adonitol & - & - & - & - & - & - \\
\hline Cellobiose & + & + & - & - & - & - \\
\hline D-Fructose & + & + & + & + & + & + \\
\hline D-Glucose & + & + & + & + & + & + \\
\hline D-Mannose & + & + & + & + & + \\
\hline Maltose & + & + & + & + & + \\
\hline D-Ribose & + & + & + & + & + \\
\hline L-Rhamnose & + & + & + & + & + \\
\hline Sucrose & + & + & + & + & + & + \\
\hline Trehalose & + & - & + & + & + & + \\
\hline L-Xylose & - & - & + & + & + \\
\hline Inulin & + & + & + & - \\
\hline
\end{tabular}

Carbon source utilization 


\begin{tabular}{|c|c|c|c|c|c|c|c|}
\hline L-Arabinose & - & - & + & - & - & - & - \\
\hline D-Arabinose & - & - & + & - & - & - & - \\
\hline D-Ribose & + & + & + & + & - & + & + \\
\hline D-Xylose & - & - & - & - & + & - & - \\
\hline L-Xylose & - & - & - & - & + & - & - \\
\hline D-Fructose & + & + & + & + & - & + & + \\
\hline D-Galactose & + & - & - & + & + & - & - \\
\hline D-Mannose & + & + & - & + & - & + & + \\
\hline L-Rhamnose & + & - & - & - & + & - & - \\
\hline Cellobiose & + & + & - & + & + & - & + \\
\hline D-Lactose & + & - & + & - & - & - & - \\
\hline Maltose & + & + & - & + & - & + & + \\
\hline L-Fucose & - & - & - & - & - & - & + \\
\hline Melibiose & - & - & + & - & + & - & - \\
\hline Trehalose & + & + & + & + & - & + & + \\
\hline Melezitose & - & - & - & - & - & - & + \\
\hline Raffinose & + & + & - & + & + & - & - \\
\hline Cellulose & + & - & - & + & - & - & - \\
\hline Inulin & - & - & - & - & + & - & - \\
\hline Starch & + & + & + & + & - & + & + \\
\hline Amygdalin & + & + & + & + & - & + & - \\
\hline Arbutin & + & + & - & + & + & + & - \\
\hline Salicin & - & - & ND & + & - & - & + \\
\hline Glycogen & + & + & - & + & + & - & - \\
\hline myo-Inositol & - & - & - & - & + & - & - \\
\hline Dulcitol & - & - & - & - & + & - & - \\
\hline Erythritol & + & - & ND & + & + & + & - \\
\hline D-Mannitol & - & - & - & + & - & + & + \\
\hline D-Sorbitol & + & - & + & - & + & - & - \\
\hline Xylitol & - & - & - & - & - & - & - \\
\hline Methyl a-D-mannoside & + & + & - & - & - & - & + \\
\hline Methyl a-D-glucoside & + & - & - & + & - & + & + \\
\hline Methyl a-D-galactoside & - & - & - & - & + & - & - \\
\hline Methyl b-D-galactoside & - & - & - & - & + & - & - \\
\hline a-Ketoglutaric acid & - & - & - & - & + & - & - \\
\hline Citric acid & + & + & - & + & + & - & - \\
\hline Fumaric acid & - & - & - & - & + & - & - \\
\hline c-Glucuronic acid & + & - & - & + & + & - & - \\
\hline Lactic acid & + & - & - & - & + & - & - \\
\hline L-Malic acid & - & - & - & - & + & - & - \\
\hline Valeric acid & - & + & ND & - & + & - & - \\
\hline Sodium acetate & + & + & - & + & + & - & - \\
\hline 5-Ketogluconate & - & - & - & - & + & - & - \\
\hline
\end{tabular}




\begin{tabular}{|l|c|c|c|c|c|c|}
\hline Sodium gluconate & - & - & ND & - & + & - \\
\hline Sodium fumarate & + & + & ND & - & + & - \\
\hline Sodium formate & + & + & ND & - & + & - \\
\hline Sodium succinate & - & - & ND & + & + & - \\
\hline Potassium acetate & - & - & - & + & + & - \\
\hline Sodium pyruvate & + & + & - & + & - & + \\
\hline
\end{tabular}

\section{Amino acid utilization}

\begin{tabular}{|c|c|c|c|c|c|c|c|}
\hline L-Alanine & + & + & + & + & + & - & - \\
\hline L-Arginine & + & + & + & + & + & - & - \\
\hline L-Aspartic acid & - & - & - & + & + & + & - \\
\hline L-Cysteine & + & + & - & + & - & - & - \\
\hline L-Creatinine & + & - & - & - & + & - & - \\
\hline L-Glutamic acid & + & + & + & - & + & - & - \\
\hline L-Histidine & + & + & + & + & + & - & - \\
\hline L-Isoleucine & + & + & - & + & + & + & - \\
\hline L-Leucine & + & + & - & + & + & - & + \\
\hline L-Serine & + & - & + & + & + & + & - \\
\hline L-Threonine & + & + & + & + & + & - & - \\
\hline L-Proline & + & + & + & + & + & - & - \\
\hline L-Tryptophan & + & + & $\mathrm{ND}$ & - & + & - & - \\
\hline L-Tyrosine & + & + & ND & + & + & - & + \\
\hline L-Valine & + & + & $\mathrm{ND}$ & + & + & - & - \\
\hline
\end{tabular}

\section{Antibiotic tolerance $(\mu \mathrm{g})$}

\begin{tabular}{|c|c|c|c|c|c|c|c|}
\hline Amikacin (30) & S & S & S & S & $\mathrm{R}$ & S & S \\
\hline Amoxicillin (30) & S & S & S & S & $\mathrm{R}$ & $\mathrm{S}$ & $S$ \\
\hline Clindamycin (25) & $S$ & $S$ & $S$ & $S$ & $\mathrm{R}$ & $S$ & $\mathrm{R}$ \\
\hline Doxycycline (25) & $S$ & $S$ & $\mathrm{R}$ & $S$ & $\mathrm{R}$ & $S$ & $S$ \\
\hline Co-trimoxazole (25) & $S$ & $S$ & $S$ & $\mathrm{~S}$ & $\mathrm{R}$ & $S$ & $S$ \\
\hline Norfloxacin (10) & $S$ & $S$ & $\mathrm{R}$ & $\mathrm{S}$ & $\mathrm{R}$ & $S$ & $S$ \\
\hline Nitrofurantoin (300) & $S$ & $S$ & $S$ & $S$ & $\mathrm{R}$ & $S$ & $S$ \\
\hline Sulfamethoxazole (50) & $S$ & $S$ & $\mathrm{R}$ & $\mathrm{R}$ & $\mathrm{R}$ & $S$ & $S$ \\
\hline PeptidoglycanD & Lys-Gly & ND & Lys-Gly & Lys-Asp & Lys-Gly & Lys-Gly & Lys-Gly \\
\hline
\end{tabular}


Strains: 1, Exiguobacterium sp. HA2; 2, E. antarcticum DSM 14480T; 3, E. sibiricum DSM 17290T; 4, E. undae DSM 14481T; 5, E. indicum IAM 15368T; 6, E. oxidotolerans JCM 12280T; 7, E. acetylicum DSM 20416T. +, Positive; 2, negative; ND, not determined; W, weakly positive; R, resistant; S, sensitive; DPG, diphosphatidylglycerol; PE, phosphatidylethanolamine; PG, phosphatidylglycerol; PI, phosphatidylinositol; PS, phosphatidylserine Data belonging to strains 2 to 6 obtained from Rodrigues et al. (2006) [20] 


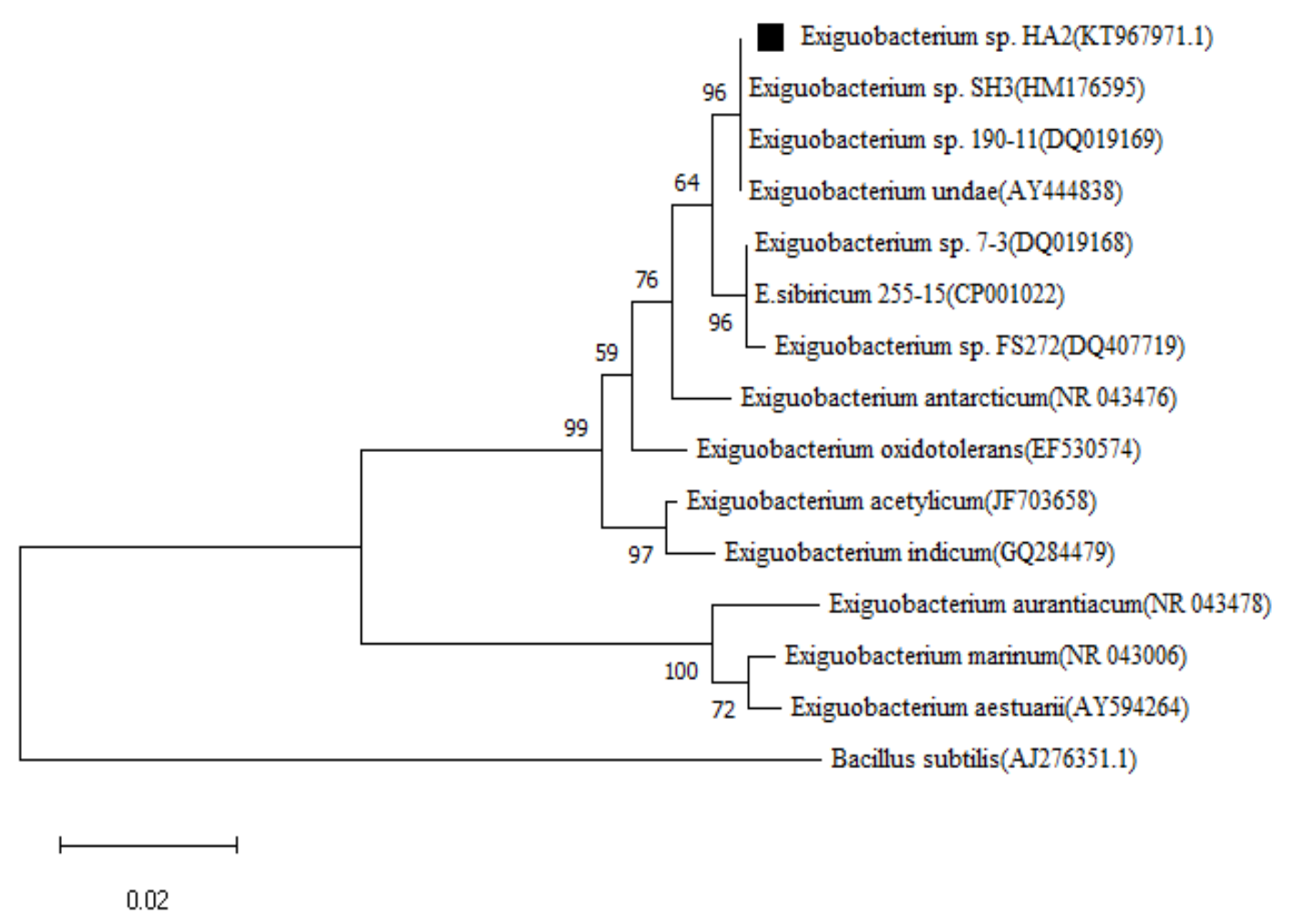

Fig. 1. Phylogenetic tree based on $16 \mathrm{~S}$ rRNA gene sequence. Numbers at the nodes indicate the bootstrap values on neighbour joining analysis 

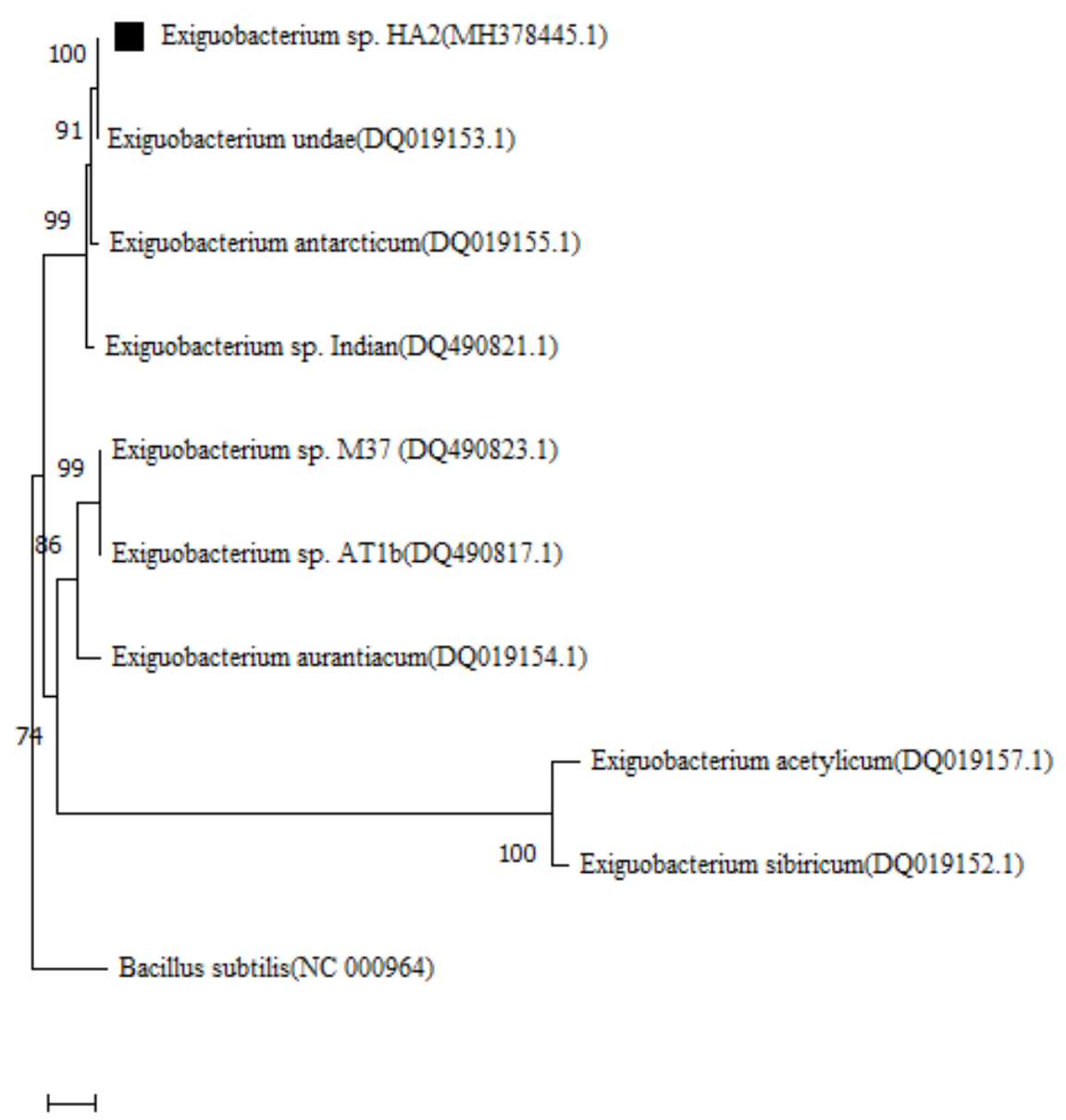

0.10

Fig. 2. Phylogenetic tree based on gyrB gene sequence. Numbers at the nodes indicate the bootstrap values on neighbour joining analysis 




Fig. 3. Phylogenetic tree based on cit $\mathrm{C}$ gene sequence. Numbers at the nodes indicate the bootstrap values on neighbour joining analysis 


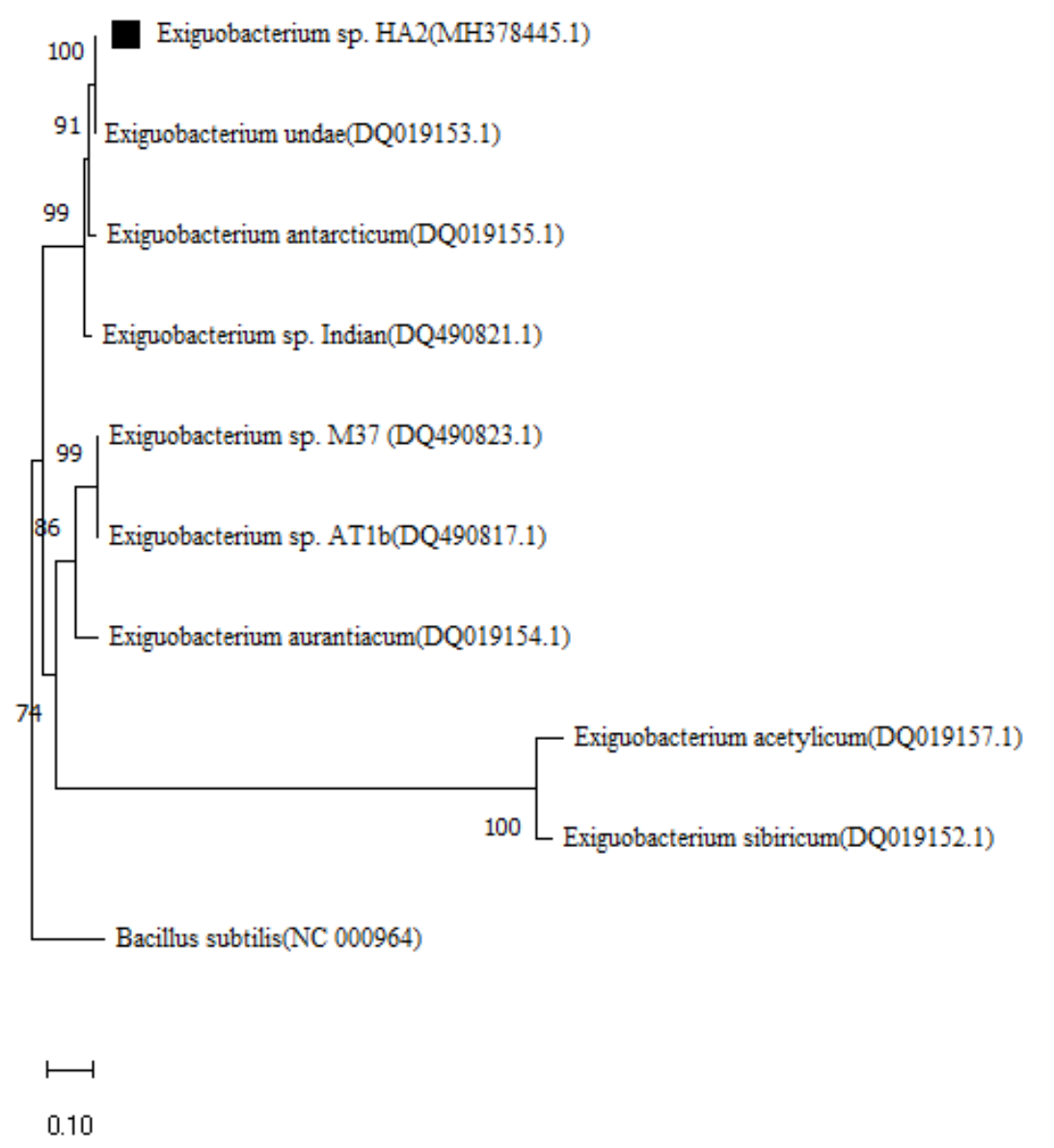

Fig. 4. Phylogenetic tree based on rpoB gene sequence. Numbers at the nodes indicate the bootstrap values on neighbour joining analysis 


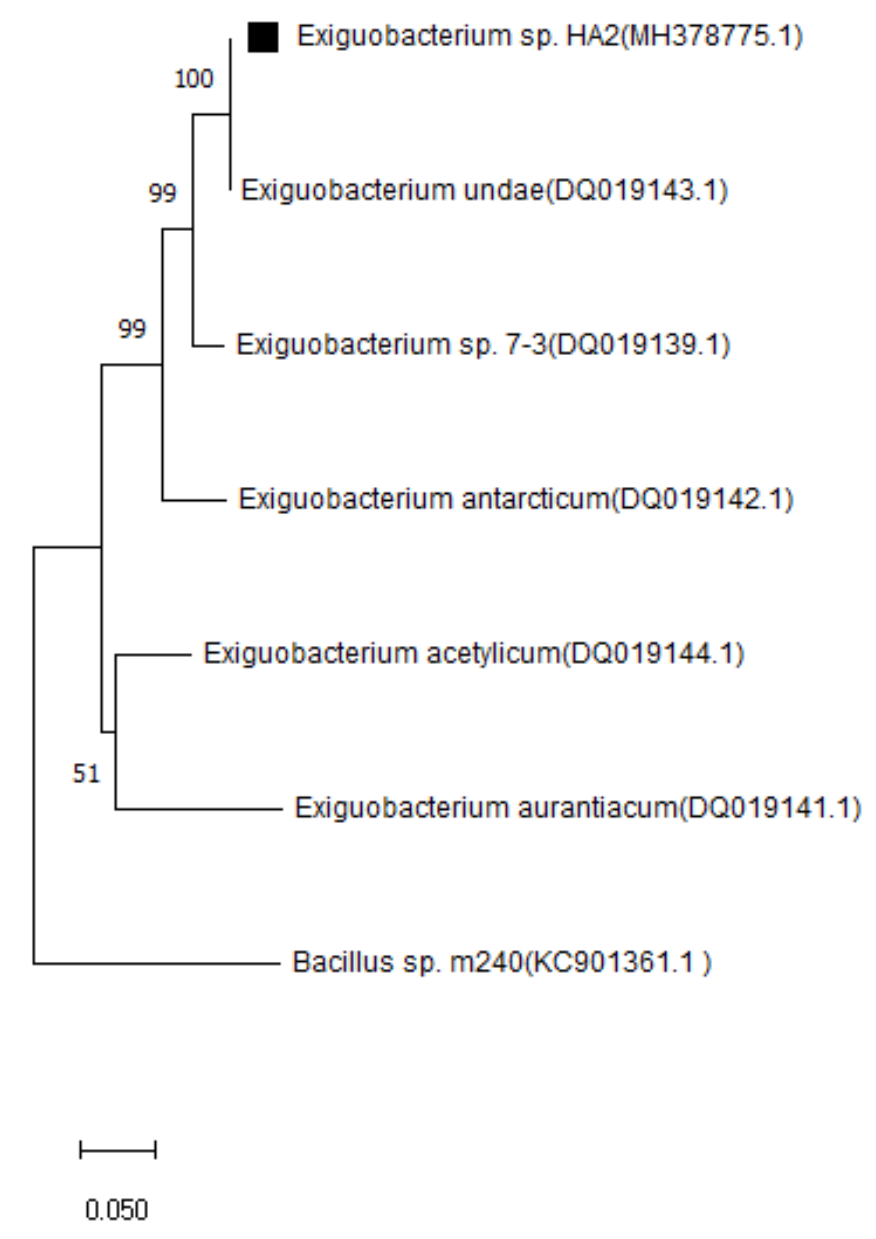

Fig. 5. Phylogenetic tree based on $h s p 70$ gene sequence. Numbers at the nodes indicate the bootstrap values on neighbour joining analysis 


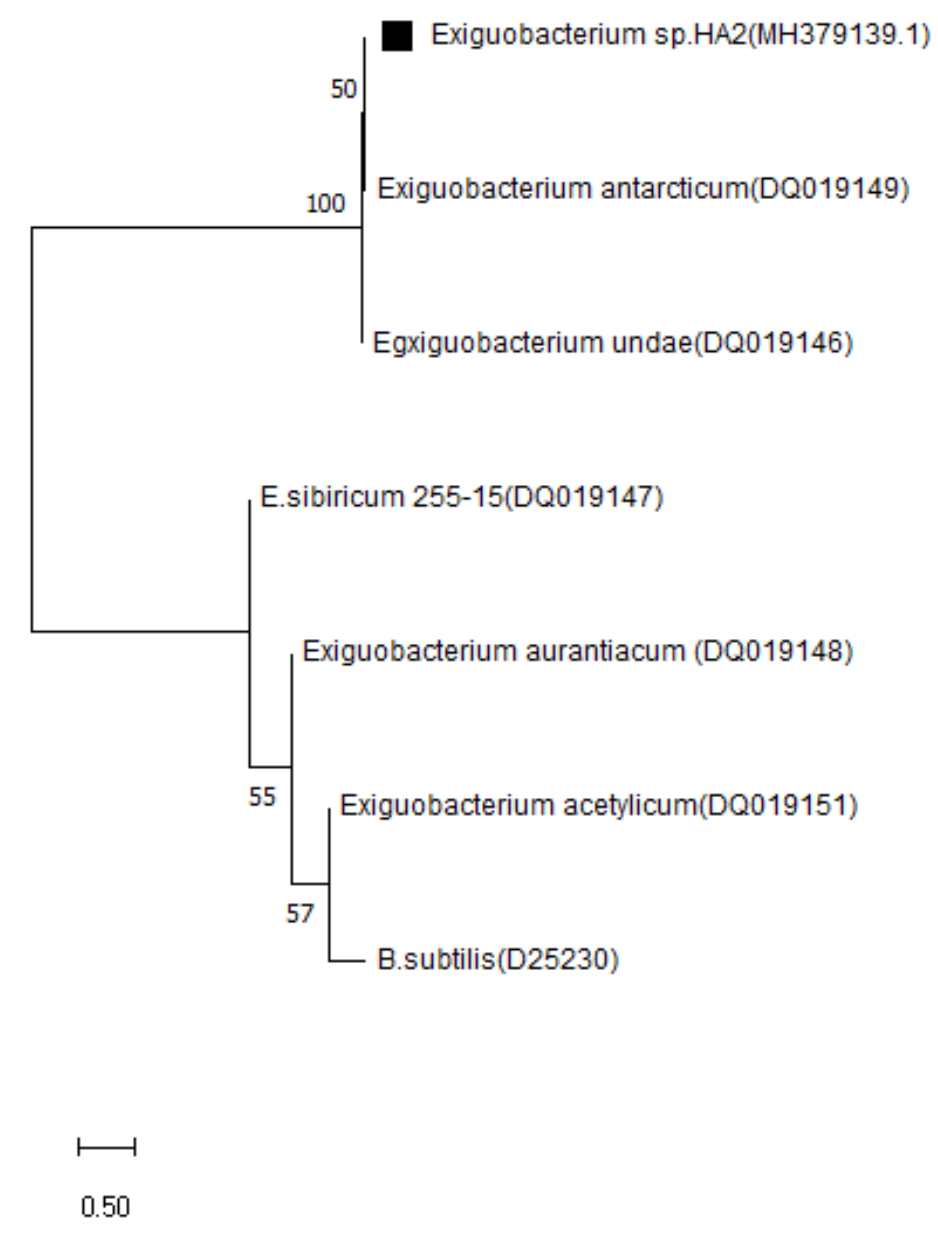

Fig. 6. Phylogenetic tree based on $c s p$ gene sequence. Numbers at the nodes indicate the bootstrap values on neighbour joining analysis 


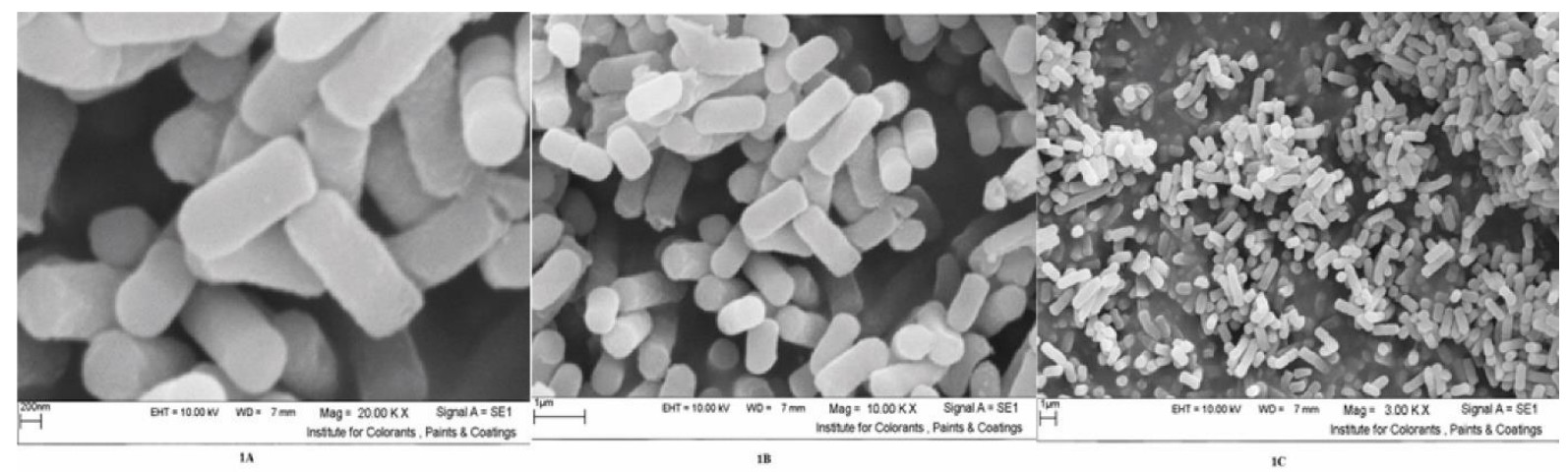

Fig. 7. Scanning electron microscopy images of Exiguobacterium sp. HA2 with different resolutions 